\title{
COMPATIBILIDADE FÍSICO-QUÍMICA DE DIFERENTES DOSES E MISTURAS DE HERBICIDAS
}

\section{PHYSICAL-CHEMICAL COMPATIBILITY OF DIFFERENT DOSES AND MIXTURES OF HERBICIDES}

Lilian Lúcia Costa ${ }^{\mathrm{a}}$, Thiago Camargo de Melo Santos ${ }^{\mathrm{b}}$, Dieimisson Paulo de Almeida ${ }^{\mathrm{c}}$, Marcelo da Costa Ferreira ${ }^{\mathrm{d}}$, Érica Fernandes Leão-Araújo ${ }^{\mathrm{a}}$, Paulo Cesar Timossi ${ }^{\mathrm{e}}$

${ }^{a}$ Departamento de Agropecuária, Instituto Federal Goiano, Goiás, Brasil. ${ }^{b}$ Louis Dreyfus Company, Mato Grosso, Brasil. ${ }^{\mathrm{c}}$ Departamento de Agricultura, Instituto de Ciência e Tecnologia COMIGO, Goiás, Brasil. 'Departamento de Ciências da Produção Agrícola, Campus de Jaboticabal/UNESP, São Paulo, Brasil. 'eUnidade Acadêmica de Ciências Agrárias, Universidade Federal de Jataí, Goiás, Brasil.

*Autor correspondente: erica.leao@ifgoiano.edu.br.

\section{INFORMAÇÕES DO ARTIGO}

\section{Histórico do artigo:}

Recebido: 14 Abril 2020.

Aceito: 13 Outubro 2020.

Publicado: 10 Dezembro 2020.

\section{Palavras-chave/Keywords:}

Atrazine/ Atrazine.

Glyphosate/ Glyphosate.

Incompatibilidade/ Incompatibility.

Hidrólise alcalina/ Alkaline hydrolysis.

Tecnologia de aplicação/ Application technology.

Direito Autoral: Este é um artigo de acesso aberto distribuído sob os termos da Licença Creative Commons, que permite uso, distribuição e reprodução irrestritos em qualquer meio, desde que $\mathrm{o}$ autor e a fonte originais sejam creditados.

\section{Citação deste artigo:}

COSTA, L. L.; SANTOS, T. C. M.; ALMEIDA, D. P.; FERREIRA, M. C.; LEÃO-ARAÚJO, É. F.; TIMOSSI, P. C. Compatibilidade físico-química de diferentes doses e misturas de herbicidas. Revista Brasileira de Herbicidas, v. 19, n. 3. 2020.

\begin{abstract}
RESUMO
A manutenção da produtividade de cultivos comerciais é dependente do uso de produtos fitossanitários, mas são restritas as informações sobre a interação entre eles quando usados em mistura. Assim, objetivou-se avaliar as propriedades físico-químicas, a tensão superficial, o ângulo de contato e o espectro de gotas de caldas fitossanitárias contendo diferentes doses e misturas dos herbicidas Glyphosate e Atrazine. Os tratamentos foram constituídos pela combinação de uma formulação do herbicida glyphosate com dois produtos comerciais formulados com o herbicida atrazine. As caldas foram avaliadas pelo método estático de compatibilidade físico-química obtendo-se tabelas descritivas quanto aos aspectos de homogeneidade/heterogeneidade. As medições da tensão superficial e ângulo de contato foram realizados durante um minuto empregando o método da gota pendente para determinar a cinética da tensão superficial e o método da gota séssil para determinar o ângulo de contato. Os atributos avaliados do espectro de gotas produzidos por cada tratamento foram o diâmetro mediano volumétrico, o coeficiente de uniformidade e a porcentagem de volume em gotas com diâmetros menores que $100 \mu \mathrm{m}$. É fundamental a agitação constante da calda fitossanitária antes e durante a aplicação para diminuir problemas de incompatibilidade entre os produtos. O espectro de gotas pode variar de acordo com a composição da calda de pulverização e com a dose do produto utilizado.
\end{abstract}


L. L. COSTA et al.

\section{Introdução}

No método de produção usando a sucessão de culturas, os restos de grãos na lavoura são comuns, devido a fatores naturais da planta como a deiscência natural das vagens e durante o processo de colheita, dando origem a plantas voluntárias na cultura subsequente, também conhecida como plantas guaxas ou tigueras (COSTA et al., 2014; MARCA et al., 2015; PETTER et al., 2015).

Para evitar interferência na cultura de interesse, uma prática eficiente é o controle químico (DAN et al., 2011; GRIGOLLI; GITTI; LOURENÇÃO, 2017), sendo comum a combinação de diferentes herbicidas com espectro sobre essas espécies. A associação dos herbicidas atrazina e glifosato, promove e intensifica o controle de plantas de folha larga com custo reduzido (TAKANO et al., 2013).

De acordo com Gazziero (2015), a mistura de produtos fitossanitários no tanque do pulverizador é uma prática usual por produtores de todas as regiões do Brasil, representando $97 \%$ dos casos. A decisão em utilizar a mistura de produtos fitossanitários no tanque do pulverizador pode ocasionar resultados inesperados como sinergismo, adição ou antagonismo devido às incompatibilidades físico-químicas, podendo melhorar o controle ou ocasionar perdas ou diminuição na eficácia da aplicação (QUEIROZ; MARTINS; CUNHA, 2008; PETTER et al., 2013; IKEDA, 2013).

Reações de incompatibilidade em tanque podem resultar na separação de fases e complexação com possível formação de aglomerados e precipitados. Essas alterações podem ocasionar dentre outros efeitos, o entupimento dos bicos de pulverizações e filtros com excessivas paradas para desentupimento durante as aplicações além de perda na eficácia dos produtos fitossanitários pela redução da quantidade de ingrediente ativo que não é aplicada junto com as gotas pulverizadas (PETTER et al., 2012).

A mensuração do $\mathrm{pH}$ também é importante para avaliação da estabilidade e solubilidade das caldas fitossanitárias. $\mathrm{O}$ pH define o grau de alcalinidade ou acidez de uma solução, numa escala de 0 a 14 . Os produtos fitossanitários podem ser degradados facilmente por hidrólise em meio alcalino, uma vez que grande parte das formulações são preparadas em água (PETTER et al., 2013).

$\mathrm{O}$ espectro de gotas, a tensão superficial e o ângulo de contato são parâmetros da tecnologia de aplicação que também devem ser observados quando se fazem misturas de produtos fitossanitários, pois estão diretamente relacionados com o controle do alvo e o resultado final da aplicação pode ser diferente do esperado em função de modificações desses parâmetros causados pela incompatibilidade físico-química da mistura (XU et al., 2011; DECARO JUNIOR et al., 2014; DECARO JÚNIOR; FERREIRA; LASMAR ,2015).

Visto a importância do conhecimento sobre as misturas de produtos fitossanitários bem como as consequências na aplicação, estudos prévios devem ser realizados a fim de evitar prejuízos na aplicação, consequentemente no controle do alvo. Com este estudo propõe-se avaliar as propriedades físico-químicas, a tensão superficial, o ângulo de contato e o espectro de gotas de caldas fitossanitárias contendo diferentes doses e misturas dos herbicidas Glyphosate e Atrazine.

\section{Material e Métodos}

Os experimentos foram conduzidos no laboratório NEDTA - Núcleo de Estudo e Desenvolvimento em Tecnologia de Aplicação, pertencente ao Departamento de Fitossanidade, da Universidade Estadual Paulista, Câmpus de Jaboticabal, SP no ano de 2019.

Os tratamentos foram constituídos pela combinação dos herbicidas glyphosate (Roundup Transorb ${ }^{\circledR}$, SL, $480 \mathrm{~g}$ e.a. $\mathrm{L}^{-1}$ Bayer) com atrazine (Primóleo ${ }^{\circledR}, \mathrm{SC}, 400$ g i.a. $\mathrm{L}^{-1}$, Syngenta) e $\operatorname{Proof}^{\circledR}$ SC (500 g i.a. $\mathrm{L}^{-1}$, de atrazina) (Syngenta) nas dosagens descritas na Tabela 1.

Tabela 1. Tratamentos utilizados para avaliações de espectro de gotas e compatibilidade físico-químicas de diferentes doses e misturas de herbicidas. Morrinhos, GO, 2019.

\begin{tabular}{cccc}
\hline & TRATAMENTOS & & \\
\hline Ingrediente ativo (i.a) & Produto comercial (p.c) & Dose (p.c L ha $\left.{ }^{-1}\right)$ & Dose $\left(\mathrm{e} . \mathrm{a} \mathrm{e} \mathrm{i.a} \mathrm{kg} \mathrm{ha}^{-1}\right)$ \\
\hline Glyphosate + Atrazine & Roundup Transorb + Primóleo & $2,0+6,0$ & $0,960+2,4$ \\
Glyphosate + Atrazine & Roundup Transorb + Primóleo & $2,0+3,0$ & $0,960+1,2$ \\
Glyphosate + Atrazine & Roundup Transorb + Proof & $2,0+4,8$ & $0,960+2,4$ \\
Glyphosate + Atrazine & Roundup Transorb + Proof & $2,0+2,4$ & $0,960+1,2$ \\
Atrazine & Primóleo & 6,0 & 2,4 \\
Atrazine & Primóleo & 3,0 & 1,2 \\
Atrazine & Proof & 4,8 & 2,4 \\
Atrazine & Proof & 2,4 & 1,2 \\
Glyphosate & Roundup Transorb & 2,0 & 0,960 \\
\hline
\end{tabular}

\section{Compatibilidade físico-química das caldas}

As avaliações de compatibilidade físico-química seguiram metodologia descrita na NBR 13875 e NBR 13074
(ABNT, 2014). Foram utilizadas três provetas de $250 \mathrm{~mL}$ para cada tratamento. No preparo das caldas utilizou-se água -padrão com dureza total de $20 \mathrm{mg} \mathrm{kg}^{-1}$ em equivalente de Carbonato de Cálcio - $\mathrm{CaCO}_{3}$. De acordo com as doses 
utilizadas, a concentração dos produtos na calda foi equivalente ao volume de aplicação de $65 \mathrm{~L} \mathrm{ha}^{-1}$. As doses dos produtos foram medidas com pipetas graduadas em quantidades ajustadas para o volume de $250 \mathrm{~mL}$ da proveta.

Adicionou-se $210 \mathrm{~mL}$ de água-padrão previamente preparada em cada proveta graduada. Em seguida, adicionou -se o respectivo herbicida na proveta e a mesma foi vertida por 10 vezes, sendo uma vez a cada 2 segundos para homogeneização. As misturas entre os produtos na calda foram feitas considerando a seguinte ordem de adição para formulações líquidas: SC (suspensão concentrada), SL (concentrado solúvel) e EC (concentrado emulsionável). Posteriormente, completou-se o volume da proveta com água-padrão até a marca de $250 \mathrm{~mL}$, sendo a proveta tampada e vertida por mais 10 vezes.

Em temperatura ambiente de $25{ }^{0} \mathrm{C} \pm 0,5$, as caldas foram avaliadas pelo método estático de compatibilidade físico-química obtendo-se tabelas descritivas quanto aos aspectos de homogeneidade/heterogeneidade. A avaliação visual da mistura quanto aos aspectos de homogeneidade/ heterogeneidade, sendo eles: floculação; sedimentação; separação de fases; formação de grumos; separação de óleo; formação de cristais; creme. As caldas foram avaliadas em quatro momentos: 1. Imediatamente após o preparo; 2. Após $2 \mathrm{~h}$ em repouso; 3. Após $6 \mathrm{~h}$ em repouso; 4. Após 24 h em repouso.

Mediu-se o potencial hidrogeniônico imediatamente após o preparo de cada calda e após o repouso nos demais momentos de avaliação. Para isto, utilizou-se um peagâmetro portátil (Kasvi), o qual foi previamente calibrado por meio de soluções padrão.

Obtiveram-se tabelas descritivas quanto à compatibilidade físico-química (aspectos de homogeneidade/heterogeneidade) e do potencial hidrogeniônico das associações de produtos nos quatro momentos de avaliação.

\section{Tensão Superficial e Ângulo de Contato}

Nas avaliações de tensão superficial e ângulo de contato utilizou-se o delineamento inteiramente casualizado com nove tratamentos e quatro repetições (Tabela 1). Foi utilizada a água como tratamento adicional. Cada unidade experimental foi constituída por uma gota.

As gotas constituídas pelas caldas herbicidas foram formadas com o auxílio de microsseringa graduada com capacidade para $500 \mu \mathrm{L}$, dispensando-se volumes de aproximadamente $5 \mu \mathrm{L}$ para cada repetição.

As medições da tensão superficial e ângulo de contato de cada tratamento foram realizadas a cada segundo durante um minuto, por meio de um tensiômetro automático equipado com câmera digital de alta velocidade e definição e, o software SCA20, utilizado para a automação do equipamento e avaliação das imagens obtidas, empregando o método da gota pendente para determinar a cinética da tensão superficial e o método da gota séssil para determinar o ângulo de contato.

\section{Espectro de gotas}

O delineamento experimental utilizado foi $\mathrm{o}$ inteiramente casualizado e doze repetições. Os tratamentos foram constituídos pela combinação dos herbicidas Glifosato (Roundup Transorb ${ }^{\circledR}$ ) e duas atrazinas (Primóleo ${ }^{\circledR}$ e Proof $\left.f^{(\mathbb{R}}\right)$ nas dosagens descritas na Tabela 1 pulverizados com o modelo de ponta AD-IA 007 (Magnojet) na pressão de $45 \mathrm{lbf} \mathrm{pol}^{-2}$ e volume ajustado para $65 \mathrm{~L} \mathrm{ha}^{-1}$.

Os parâmetros avaliados do espectro de gotas produzidos por cada calda fitossanitária foram o diâmetro mediano volumétrico (Dv 0,5), o coeficiente de uniformidade das gotas pulverizadas (Coef. Unif.) e a porcentagem de volume em gotas com diâmetros menores que $100 \mu \mathrm{m}(\%<100 \mu \mathrm{m})$.

O diâmetro das gotas foi determinado por difração de raio laser durante a passagem das gotas pulverizadas pela região de amostragem de um analisador de tamanho de partículas (Mastersizer, Malvern Instruments Limited), ajustado para avaliar gotas de 0,5 a $900 \mu \mathrm{m}$.

O coeficiente de uniformidade foi obtido por meio da equação 1: Coef. Unif. $=($ Dv0.9-Dv0,1)/Dv0,5. Em que: Coef. Unif. $=$ Coeficiente de Uniformidade (adimensional); Dv0,1 e Dv0,9 referem-se ao diâmetro da gota $(\mu \mathrm{m})$, tal que 10 e $90 \%$, respectivamente, do volume aspergido é composto por gotas de diâmetro inferior a ele; Dv 0,5 refere -se ao diâmetro mediano volumétrico.

A decodificação dos dados, segundo o algoritmo elaborado para a caracterização do diâmetro das gotas por difração de raio laser foram processados e tabelados diretamente pelo programa Mastersizer $\mathrm{S}^{\circledR}$, versão 2.19.

\section{Análise estatística}

Os dados obtidos, após confirmação dos pressupostos de normalidade do resíduo (Shapiro-Wilk) e homogeneidade de variâncias (Cochran), foram submetidos ao teste $\mathrm{F}$ da análise de variância e, quando significativo $(\mathrm{p}<0,01$ ou $\mathrm{p}<0,05)$, as médias dos tratamentos foram comparadas pelo teste de Scott-Knott.

Para análise estatística dos dados de tensão superficial e ângulo de contato das caldas foram considerados apenas os dados obtidos no tempo de sessenta segundos quando os valores referentes a estes parâmetros já se estabilizaram e caracteriza o final da avaliação da cinética da tensão superficial e ângulo de contato de cada tratamento.

\section{Resultados e Discussão}

\section{Compatibilidade físico-química das caldas}

Houve sedimentação e formação de grumo dos herbicidas Primóleo ${ }^{\circledR}$ e Proof ${ }^{\circledR}$ utilizados sozinhos ou em mistura com o Roundup Transorb ${ }^{\circledR}$ imediatamente após o preparo das caldas e após duas, seis e 24 horas após o preparo da calda (HAP) (Tabela 2). O herbicida Roundup Transorb $^{\circledR}$ utilizado somente com a água manteve-se estável para todos os parâmetros, independente da agitação, em todos os períodos de avaliação (Tabela 2). 
L. L. COSTA et al.

Tabela 2. Parâmetros avaliados para o estudo de compatibilidade físico-química das misturas de herbicidas. Morrinhos, GO, 2019.

\begin{tabular}{|c|c|c|c|c|c|c|}
\hline \multirow{2}{*}{$\begin{array}{c}\text { Produto Comercial } \\
\text { Herbicidas }\end{array}$} & \multirow[b]{2}{*}{ Doses (p.c.) } & \multicolumn{5}{|c|}{ Imediatamente após o preparo } \\
\hline & & ${ }^{2}$ Floc. & Sed. & S. Fases & Grumos & Creme \\
\hline${ }^{1}$ RT + Primóleo ${ }^{\circledR}$ & $2,0+6,0 \mathrm{~L} \mathrm{ha}^{-1}$ & $*$ & $\mathrm{P}$ & $*$ & $\mathrm{P}$ & $*$ \\
\hline $\mathrm{RT}+$ Primóleo $^{\circledR}$ & $2,0+3,0 \mathrm{~L} \mathrm{ha}^{-1}$ & $*$ & $\mathrm{P}$ & $*$ & $\mathrm{P}$ & * \\
\hline RT $+\operatorname{Proof}^{\circledR}$ & $2,0+4,8 \mathrm{~L} \mathrm{ha}^{-1}$ & $*$ & $\mathrm{P}$ & $*$ & $\mathrm{P}$ & $*$ \\
\hline RT + Proof $^{(\mathbb{R}}$ & $2,0+2,4 \mathrm{~L} \mathrm{ha}^{-1}$ & $*$ & $\mathrm{P}$ & $*$ & $\mathrm{P}$ & $*$ \\
\hline Primóleo $^{\circledR}$ & $6,0 \mathrm{~L} \mathrm{ha}^{-1}$ & $*$ & $\mathrm{P}$ & $*$ & $\mathrm{P}$ & $*$ \\
\hline Primóleo $^{\circledR}$ & $3,0 \mathrm{~L} \mathrm{ha}^{-1}$ & $*$ & $\mathrm{P}$ & * & $\mathrm{P}$ & $*$ \\
\hline $\operatorname{Proof}^{(\mathbb{B}}$ & $4,8 \mathrm{~L} \mathrm{ha}^{-1}$ & * & $\mathrm{P}$ & $*$ & $\mathrm{P}$ & $*$ \\
\hline Proof $^{\circledR}$ & $2,4 \mathrm{~L} \mathrm{ha}^{-1}$ & $*$ & $\mathrm{P}$ & $*$ & $\mathrm{P}$ & $*$ \\
\hline \multirow[t]{2}{*}{$\mathrm{RT}$} & $2,0 \mathrm{~L} \mathrm{ha}^{-1}$ & $*$ & $*$ & $*$ & $*$ & $*$ \\
\hline & & \multicolumn{5}{|c|}{ Duas horas após o preparo } \\
\hline Herbicidas & Doses (p.c.) & ${ }^{2}$ Floc. & Sed. & S. Fases & Grumos & Creme \\
\hline${ }^{1} \mathrm{RT}+$ Primóleo ${ }^{\circledR}$ & $2,0+6,0 \mathrm{~L} \mathrm{ha}^{-1}$ & $*$ & $\mathrm{P}$ & $*$ & $\mathrm{P}$ & $\mathrm{P}$ \\
\hline RT + Primóleo ${ }^{\circledR}$ & $2,0+3,0 \mathrm{~L} \mathrm{ha}^{-1}$ & $*$ & $\mathrm{P}$ & $*$ & $\mathrm{P}$ & $P$ \\
\hline RT + Proof $^{\circledR}$ & $2,0+4,8 \mathrm{~L} \mathrm{ha}^{-1}$ & $\mathrm{P}$ & $\mathrm{P}$ & $\mathrm{P}$ & $\mathrm{P}$ & $P$ \\
\hline RT + Proof $^{(\mathbb{R}}$ & $2,0+2,4 \mathrm{~L} \mathrm{ha}^{-1}$ & $\mathrm{P}$ & $\mathrm{P}$ & $\mathrm{P}$ & $\mathrm{P}$ & $\mathrm{P}$ \\
\hline Primóleo $^{\circledR}$ & $6,0 \mathrm{~L} \mathrm{ha}^{-1}$ & $*$ & $\mathrm{P}$ & $*$ & $\mathrm{P}$ & $*$ \\
\hline Primóleo $^{\circledR}$ & $3,0 \mathrm{~L} \mathrm{ha}^{-1}$ & $*$ & $\mathrm{P}$ & $*$ & $\mathrm{P}$ & $*$ \\
\hline $\operatorname{Proof}^{\circledR}$ & $4,8 \mathrm{~L} \mathrm{ha}^{-1}$ & $*$ & $\mathrm{P}$ & $*$ & $\mathrm{P}$ & $*$ \\
\hline Proof $^{(B)}$ & $2,4 \mathrm{~L} \mathrm{ha}^{-1}$ & $*$ & $\mathrm{P}$ & $*$ & $\mathrm{P}$ & $*$ \\
\hline \multirow[t]{2}{*}{$\mathrm{RT}$} & $2,0 \mathrm{~L} \mathrm{ha}^{-1}$ & * & $*$ & * & * & $*$ \\
\hline & & \multicolumn{5}{|c|}{ Seis horas após o preparo } \\
\hline Herbicidas & Doses (p.c.) & ${ }^{2}$ Floc. & Sed. & S. Fases & Grumos & Creme \\
\hline${ }^{1} \mathrm{RT}+$ Primóleo $^{\mathbb{B}}$ & $2,0+6,0 \mathrm{~L} \mathrm{ha}^{-1}$ & $*$ & $\mathrm{P}$ & * & $\mathrm{P}$ & $\mathrm{P}$ \\
\hline $\mathrm{RT}+$ Primóleo $^{\circledR}$ & $2,0+3,0 \mathrm{~L} \mathrm{ha}^{-1}$ & $*$ & $P$ & $*$ & $P$ & $P$ \\
\hline RT + Proof $^{\mathbb{R}}$ & $2,0+4,8 \mathrm{~L} \mathrm{ha}^{-1}$ & $\mathrm{P}$ & $\mathrm{P}$ & $\mathrm{P}$ & $P$ & $\mathrm{P}$ \\
\hline RT + Proof $^{\mathbb{R}}$ & $2,0+2,4 \mathrm{~L} \mathrm{ha}^{-1}$ & $\mathrm{P}$ & $\mathrm{P}$ & $\mathrm{P}$ & $\mathrm{P}$ & $\mathrm{P}$ \\
\hline Primóleo $^{\circledR}$ & $6,0 \mathrm{~L} \mathrm{ha}^{-1}$ & $*$ & $\mathrm{P}$ & $*$ & $\mathrm{P}$ & $\mathrm{P}$ \\
\hline Primóleo $^{\circledR}$ & $3,0 \mathrm{~L} \mathrm{ha}^{-1}$ & $*$ & $\mathrm{P}$ & * & $\mathrm{P}$ & $\mathrm{P}$ \\
\hline Proof $^{(B)}$ & $4,8 \mathrm{~L} \mathrm{ha}^{-1}$ & $*$ & $\mathrm{P}$ & $*$ & $\mathrm{P}$ & $\mathrm{P}$ \\
\hline Proof $^{(B)}$ & $2,4 \mathrm{~L} \mathrm{ha}^{-1}$ & $*$ & $\mathrm{P}$ & $*$ & $\mathrm{P}$ & $\mathrm{P}$ \\
\hline \multirow[t]{2}{*}{ RT } & $2,0 \mathrm{~L} \mathrm{ha}^{-1}$ & $*$ & $*$ & $*$ & $*$ & $*$ \\
\hline & & \multicolumn{5}{|c|}{ Vinte e quatro horas após o preparo } \\
\hline Herbicidas & Doses (p.c.) & ${ }^{2}$ Floc. & Sed. & S. Fases & Grumos & Creme \\
\hline${ }^{1}$ RT + Primóleo ${ }^{\circledR}$ & $2,0+6,0 \mathrm{~L} \mathrm{ha}^{-1}$ & * & $\mathrm{P}$ & $\mathrm{P}$ & $\mathrm{P}$ & $\mathrm{P}$ \\
\hline $\mathrm{RT}+$ Primóleo $^{\circledR}$ & $2,0+3,0 \mathrm{~L} \mathrm{ha}^{-1}$ & $*$ & $P$ & $P$ & $P$ & $P$ \\
\hline $\mathrm{RT}+\operatorname{Proof}^{\circledR}$ & $2,0+4,8 \mathrm{~L} \mathrm{ha}^{-1}$ & $P$ & $\mathrm{P}$ & $\mathrm{P}$ & $P$ & $\mathrm{P}$ \\
\hline $\mathrm{RT}+\operatorname{Proof}^{\mathbb{R}}$ & $2,0+2,4 \mathrm{~L} \mathrm{ha}^{-1}$ & $\mathrm{P}$ & $\mathrm{P}$ & $\mathrm{P}$ & $\mathrm{P}$ & $\mathrm{P}$ \\
\hline Primóleo $^{\circledR}$ & $6,0 \mathrm{~L} \mathrm{ha}^{-1}$ & $*$ & $\mathrm{P}$ & $*$ & $\mathrm{P}$ & $\mathrm{P}$ \\
\hline Primóleo $^{\circledR}$ & $3,0 \mathrm{~L} \mathrm{ha}^{-1}$ & * & $\mathrm{P}$ & $*$ & $\mathrm{P}$ & $\mathrm{P}$ \\
\hline Proof $^{(B)}$ & $4,8 \mathrm{~L} \mathrm{ha}^{-1}$ & * & $\mathrm{P}$ & $*$ & $\mathrm{P}$ & $\mathrm{P}$ \\
\hline Proof $^{(\mathbb{B}}$ & $2,4 \mathrm{~L} \mathrm{ha}^{-1}$ & $*$ & $\mathrm{P}$ & $*$ & $\mathrm{P}$ & $\mathrm{P}$ \\
\hline $\mathrm{RT}$ & $2,0 \mathrm{~L} \mathrm{ha}^{-1}$ & $*$ & $*$ & $*$ & $*$ & $*$ \\
\hline
\end{tabular}

${ }^{1}$ RT = Roundup Transorb ${ }^{\circledR} .{ }^{2}$ Floc = Floculação; Sed = Sedimentação; Sep Fas = Separação de Fases. *= Não Houve; P = Presença do parâmetro avaliado.

O processo de sedimentação de partículas em líquidos em repouso é natural. O importante é que haja redispersão destas partículas quando houver agitação contínua da calda no tanque de pulverização. Segundo Petter et al., (2013), a presença de sedimentos no fundo do tanque do pulverizador pode resultar em menor eficácia no controle 
L. L. COSTA et al.

do alvo preconizado devido a concentração desuniforme do produto na aplicação.

Nas avaliações de duas, seis e 24 HAP das caldas, verificou-se que a mistura do Roundup Transorb ${ }^{\circledR}$ com $\operatorname{Proof}^{\circledR}\left(2,4 \mathrm{~L} \mathrm{ha}^{-1}\right.$ e $\left.4,8 \mathrm{~L} \mathrm{ha}^{-1}\right)$ apresentaram floculação, sedimentação, separação de fases, formação de grumo e de creme (Tabela 2). A floculação foi observada somente nestes tratamentos. A floculação de produtos fitossanitários é decorrente de compostos insolúveis na calda formando aglomerados que ficam retidos nos filtros do circuito hidráulico ou causar o entupimento das pontas de pulverização (NICOLAI; CHRISTOFFOLETI, 2007).

A separação de fases também foi observada na avaliação de 24 HAP das caldas com as misturas de Roundup Transorb ${ }^{\circledR}$ com Primóleo $^{\circledR}\left(3,0 \mathrm{~L} \mathrm{ha}^{-1}\right.$ e $\left.6,0 \mathrm{~L} \mathrm{ha}^{-1}\right)$ (Tabela 2). A ocorrência da separação de fases indica que partículas dos herbicidas se encontravam no fundo da proveta e, similarmente, estariam no fundo do tanque do pulverizador. Sem agitação prévia, de forma a redispersar a formulação, um dos problemas que poderia incorrer seria fitotoxidade na cultura proporcionado por maior concentração dos herbicidas.

A formação de creme foi observada a partir de duas HAP das caldas nos tratamentos com Proof ${ }^{\circledR}$ e Primóleo ${ }^{\circledR}$ associados ao Roundup Transorb ${ }^{\circledR}$, e com seis e 24 HAP nos

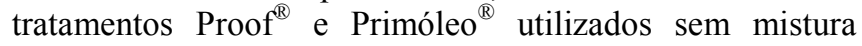

(Tabela 2). Portanto, faz-se necessário o uso de um sistema de agitação no tanque eficiente de forma que mantenha a formulação dos produtos dispersas na calda de forma homogênea.

$\mathrm{O}$ pH da calda também é um parâmetro utilizado como indicativo de incompatibilidade entre produtos. De acordo com Petter et al. (2013), quando o pH da mistura está próximo a neutralidade apresentam maiores quantidades de cátions que podem se ligar aos ativos dispersos, levando assim a ocorrência de precipitados e acelerar a degradação do produto diminuindo a quantidade de ingrediente ativo disponível que por sua vez diminuem a eficácia biológica do produto. Desta forma, a redução do $\mathrm{pH}$ reduz a hidrólise alcalina de produtos sensíveis à calda com $\mathrm{pH}$ elevado (CUNHA; ALVES, 2009).

Neste estudo, independente das diferenças estatísticas entre os tratamentos observadas, verificou-se que o herbicida Proof $^{\circledR}$ na maior ou menor dose tiveram $\mathrm{pH}$ das caldas mais próximas a neutralidade (Tabela 3). Os demais tratamentos analisados tiveram $\mathrm{pH}$ dentro de uma faixa mais adequada para caldas herbicidas. Alguns herbicidas, tais como o glifosato, têm sua eficiência elevada na planta com a redução do $\mathrm{pH}$ da água a valores próximos a 4,0 (WANAMARTA; PENNER, 1989). De acordo com Murphy (2004), a maioria dos produtos não são afetados pela acidez como o são pela alcalinidade.

Tabela 3. Valores do potencial hidrogeniônico $(\mathrm{pH})$ das misturas de herbicidas obtidos imediatamente após o preparo das caldas fitossanitárias (0 hora) e após 2, 6 e 24 horas. Morrinhos, GO, 2019.

\begin{tabular}{cccccc}
\hline & & \multicolumn{3}{c}{ Potencial Hidrogeniônico $(\mathrm{pH})$} \\
\cline { 3 - 5 } Herbicidas & Doses (p.c.) & 0 hora & 2 horas & 6 horas & 24 horas \\
\hline${ }^{1}$ RT + Primóleo & $4,61 \mathrm{ab}$ & $4,63 \mathrm{~b}$ & $4,60 \mathrm{a}$ & $4,68 \mathrm{~b}$ \\
RT + Primóleo & $2,0+6,0 \mathrm{~L} \mathrm{ha}^{\circledR}$ & $4,65 \mathrm{~b}$ & $4,62 \mathrm{~b}$ & $4,63 \mathrm{a}$ & $4,69 \mathrm{~b}$ \\
RT + Proof & $2,0+3,0 \mathrm{~L} \mathrm{ha}^{-1}$ & $4,75 \mathrm{c}$ & $4,75 \mathrm{~d}$ & $4,74 \mathrm{~b}$ & $4,86 \mathrm{~d}$ \\
RT + Proof & $2,0+4,8 \mathrm{~L} \mathrm{ha}^{-1}$ & $4,74 \mathrm{c}$ & $4,76 \mathrm{~d}$ & $4,73 \mathrm{~b}$ & $4,83 \mathrm{~d}$ \\
Primóleo $^{\circledR}$ & $2,0+2,4 \mathrm{~L} \mathrm{ha}^{-1}$ & $4,58 \mathrm{a}$ & $4,53 \mathrm{a}$ & $4,63 \mathrm{~b}$ & $4,57 \mathrm{a}$ \\
Primóleo $^{\circledR}$ & $6,0 \mathrm{~L} \mathrm{ha}^{-1}$ & $5,11 \mathrm{~d}$ & $4,99 \mathrm{e}$ & $5,15 \mathrm{c}$ & $5,20 \mathrm{e}$ \\
Proof $^{\circledR}$ & $3,0 \mathrm{~L} \mathrm{ha}^{-1}$ & $6,57 \mathrm{e}$ & $6,65 \mathrm{f}$ & $6,78 \mathrm{~d}$ & $6,65 \mathrm{f}$ \\
Proof $^{\circledR}$ & $4,8 \mathrm{~L} \mathrm{ha}^{-1}$ & $6,84 \mathrm{f}$ & $6,82 \mathrm{~g}$ & $7,21 \mathrm{e}$ & $6,91 \mathrm{~g}$ \\
RT & $2,4 \mathrm{~L} \mathrm{ha}^{-1}$ & $4,71 \mathrm{c}$ & $4,70 \mathrm{c}$ & $4,71 \mathrm{~b}$ & $4,78 \mathrm{c}$ \\
\hline
\end{tabular}

${ }^{1} \mathrm{RT}=$ Roundup Transorb $^{\circledR}$. Médias seguidas de mesma letra não diferem entre si pelo Teste de Scott-Knott a $5 \%$ de probabilidade.

\section{Tensão superficial e ângulo de contato}

A água, na sua forma pura e em estado líquido tem a tendência de formar gotas esféricas com tensão em torno de $79,0 \mathrm{mNm}^{-1}$ e ângulo de contato de $110^{\circ}$ conforme constatado no presente estudo com valores similares (Tabela 4). Os herbicidas avaliados utilizados isolados ou em mistura proporcionaram redução da tensão superficial e ângulo de contato em relação à água (Tabela 4). 
L. L. COSTA et al.

Tabela 4. Valores médios da tensão superficial $\left(\mathrm{mNm}^{-1}\right)$ e do ângulo de contato $\left(\theta^{\circ}\right)$ das diferentes caldas herbicidas analisadas no tempo de 60 segundos. Morrinhos, GO, 2019.

\begin{tabular}{|c|c|c|c|}
\hline \multicolumn{2}{|c|}{ Tratamentos } & Tensão superficial & Ângulo de contato \\
\hline${ }^{1}$ RT + Primóleo ${ }^{\circledR}$ & $2,0+6,0 \mathrm{~L} \mathrm{ha}^{-1}$ & $29,51 \mathrm{a}$ & $30,84 \mathrm{a}$ \\
\hline RT + Primóleo ${ }^{\circledR}$ & $2,0+3,0 \mathrm{~L} \mathrm{ha}^{-1}$ & $29,34 \mathrm{a}$ & $36,52 \mathrm{~b}$ \\
\hline $\mathrm{RT}+\operatorname{Proof}^{\circledR}$ & $2,0+4,8 \mathrm{~L} \mathrm{ha}^{-1}$ & $29,69 \mathrm{a}$ & $45,05 \mathrm{c}$ \\
\hline RT $+\operatorname{Proof}^{(B)}$ & $2,0+2,4 \mathrm{~L} \mathrm{ha}^{-1}$ & $29,72 \mathrm{a}$ & $56,01 \mathrm{~d}$ \\
\hline Primóleo $^{\circledR}$ & $6,0 \mathrm{~L} \mathrm{ha}^{-1}$ & $27,99 \mathrm{a}$ & $36,70 \mathrm{~b}$ \\
\hline Primóleo $^{\circledR}$ & $3,0 \mathrm{~L} \mathrm{ha}^{-1}$ & $31,44 \mathrm{a}$ & $41,32 \mathrm{c}$ \\
\hline $\operatorname{Proof}^{\mathbb{B}}$ & $4,8 \mathrm{~L} \mathrm{ha}^{-1}$ & $29,89 \mathrm{a}$ & $39,26 \mathrm{c}$ \\
\hline $\operatorname{Proof}^{\circledR}$ & $2,4 \mathrm{~L} \mathrm{ha}^{-1}$ & $30,77 \mathrm{a}$ & $43,20 \mathrm{c}$ \\
\hline Roundup Transorb ${ }^{\circledR}$ & $2,0 \mathrm{~L} \mathrm{ha}^{-1}$ & $37,55 \mathrm{a}$ & $72,35 \mathrm{e}$ \\
\hline Água & -------- & $72,03 \mathrm{~b}$ & $108,18 \mathrm{f}$ \\
\hline \multicolumn{2}{|c|}{ Teste $\mathrm{F}$} & $17,52 * *$ & $198,63 * *$ \\
\hline \multicolumn{2}{|c|}{ Coeficiente de Variação } & 18,31 & 6,50 \\
\hline
\end{tabular}

${ }^{1} \mathrm{RT}=$ Roundup Transorb ${ }^{\circledR}$. Médias seguidas de mesma letra não diferem entre si pelo Teste de Scott-Knott a $5 \%$ de probabilidade. Pelo teste $\mathrm{F},{ }^{*}$ significativo a $1 \%$ de probabilidade.

Este efeito de diminuição da tensão superficial e ângulo de contato das gotas formado normalmente possuem correlação positiva com o espalhamento da gota, ou seja, quanto menor a tensão superficial e o ângulo de contato com a superfície do alvo maior será o espalhamento e, consequentemente maior o depósito e cobertura do alvo (VAN ZYL et al., 2010; XU et al., 2011; DECARO JUNIOR et al., 2014; DECARO JÚNIOR; FERREIRA; LASMAR ,2015).

\section{Espectro de gotas}

Houve diferença significativa entre os tratamentos para diâmetro mediano volumétrico (Dv0,5) (Tabela 5). Mas independente dessas diferenças existe um padrão de classificação dos espectros das gotas produzidos pelos modelos de pontas em uma pulverização (ASABE, 2009).

Baseando-se nesta classificação e pelos valores de Dv0,5 observou-se que a combinação de Roundup Transorb $^{\circledR}+$ Primóleo $^{\circledR}$ na dose de $6 \mathrm{~L} \mathrm{ha}^{-1}$ e Roundup Transorb $^{\circledR}$ teve espectro classificado na classe média; Roundup Transorb ${ }^{\circledR}+$ Primóleo $^{\circledR}$ na dose de $3 \mathrm{~L} \mathrm{ha}^{-1}$, Primóleo ${ }^{\circledR}$ nas doses de 3 e $6 \mathrm{~L} \mathrm{ha}^{-1}$ e Proof ${ }^{\circledR}$ na dose de 2,4 $\mathrm{L} \mathrm{ha}^{-1}$ na classe grossa; Roundup Transorb ${ }^{\circledR}+$ Proof $^{\circledR}$ nas doses de 2,4 e 4,8 $\mathrm{L} \mathrm{ha}^{-1}$ na classe muito grossa e Proof ${ }^{(B)}$ na dose de $4,8 \mathrm{~L} \mathrm{ha}^{-1}$ na classe extremamente grossa (Tabela 5).

Tabela 5. Síntese da análise de variância e do teste de médias para as variáveis: diâmetro mediano volumétrico $(\mathrm{Dv} 0,5)(\mu \mathrm{m})$, porcentagem do volume pulverizado composto por gotas com diâmetro menor ou igual a 100 micrometros $(\% \leq 100 \mu \mathrm{m})$ e Coeficiente de Uniformidade (Coef. Unif.) das gotas pulverizadas por diferentes doses e misturas de herbicidas. Morrinhos, GO, 2019.

\begin{tabular}{|c|c|c|c|c|}
\hline \multicolumn{2}{|c|}{ Tratamentos } & DMV & $\% \leq 100$ & Coef. Unif. \\
\hline${ }^{1}$ RT + Primóleo ${ }^{\circledR}$ & $2,0+6,0 \mathrm{~L} \mathrm{ha}^{-1}$ & $301,38 \mathrm{e}$ & $3,96 \mathrm{c}$ & $2,08 \mathrm{c}$ \\
\hline RT + Primóleo ${ }^{\circledR}$ & $2,0+3,0 \mathrm{~L} \mathrm{ha}^{-1}$ & $395,93 \mathrm{~d}$ & $3,57 \mathrm{c}$ & $1,70 \mathrm{a}$ \\
\hline RT $+\operatorname{Proof}^{\circledR}$ & $2,0+4,8 \mathrm{~L} \mathrm{ha}^{-1}$ & $481,64 \mathrm{~b}$ & $1,19 \mathrm{a}$ & $1,60 \mathrm{a}$ \\
\hline $\mathrm{RT}+\operatorname{Proof}^{\mathrm{B}}$ & $2,0+2,4 \mathrm{~L} \mathrm{ha}^{-1}$ & $482,58 \mathrm{~b}$ & $2,18 \mathrm{~b}$ & $1,67 \mathrm{a}$ \\
\hline Primóleo $^{\circledR}$ & $6,0 \mathrm{~L} \mathrm{ha}^{-1}$ & $399,74 \mathrm{~d}$ & $5,13 \mathrm{~d}$ & $2,19 \mathrm{c}$ \\
\hline Primóleo $^{\circledR}$ & $3,0 \mathrm{~L} \mathrm{ha}^{-1}$ & $381,03 \mathrm{~d}$ & $6,33 \mathrm{e}$ & $1,97 \mathrm{c}$ \\
\hline Proof $^{\circledR}$ & $4,8 \mathrm{~L} \mathrm{ha}^{-1}$ & $557,40 \mathrm{a}$ & $3,76 \mathrm{c}$ & $1,87 \mathrm{~b}$ \\
\hline $\operatorname{Proof}^{\circledR}$ & $2,4 \mathrm{~L} \mathrm{ha}^{-1}$ & $391,49 \mathrm{~d}$ & $5,38 \mathrm{~d}$ & $1,83 \mathrm{~b}$ \\
\hline Roundup Transorb ${ }^{\circledR}$ & $2,0 \mathrm{~L} \mathrm{ha}^{-1}$ & $310,84 \mathrm{e}$ & $6,69 \mathrm{e}$ & $2,39 \mathrm{~d}$ \\
\hline \multicolumn{2}{|c|}{ Teste F } & $66,16^{* *}$ & $34,44 * *$ & $16,25 * *$ \\
\hline \multicolumn{2}{|c|}{ Coeficiente de Variação } & 5,99 & 20,59 & 9,66 \\
\hline
\end{tabular}

${ }^{1} \mathrm{RT}=$ Roundup Transorb ${ }^{\circledR}$. Médias seguidas de mesma letra não diferem entre si pelo Teste de Scott-Knott a $5 \%$ de probabilidade. Pelo teste $\mathrm{F},{ }^{*} *$ significativo a $1 \%$ de probabilidade. 
O modelo AD-IA na pressão de $45 \mathrm{lbf} \mathrm{pol}^{-2}$ em que foi utilizado nesse experimento, deveria produzir gotas muito grossas de acordo com a indicação do fabricante. Mas estas diferenças entre os tratamentos podem estar relacionadas com a composição da calda devido aos inertes presentes nas diferentes formulações, conforme já relatado por alguns autores (COSTA et al., 2017a;b).

As avaliações para a classificação do espectro de gotas produzidos pelos diferentes modelos de pontas disponíveis no mercado são mensurados com caldas padrão de acordo com a norma ANSI/ASAE S572.1. Desta forma, comprova-se que o espectro de gotas pode variar de acordo com a composição da calda de pulverização.

A interpretação dos dados de porcentagem do volume de gotas com diâmetro inferior a $100 \mu \mathrm{m}$ permite a estimativa do potencial de deriva da aplicação. Gotas menores que $100 \mu \mathrm{m}$ produzidas no processo de pulverização são consideradas por vários autores de alto risco de deriva (ARVIDSSON; BERGSTRÖM; KREUGER, 2011; OLIVEIRA; ANTUNIASSI; GANDOLFO, 2015). De acordo com Cunha et al. (2003), não existe valor-padrão indicativo de risco de deriva, mas sugerem que valores abaixo de $15 \%$ de volume de gotas com diâmetro inferior a $100 \mu \mathrm{m}$, em geral, são mais adequados para aplicações e ambientalmente mais seguras. Assim, independente das diferenças estatísticas observadas, todos os tratamentos apresentaram abaixo de $7 \%$ do espectro de gotas compostos por gotas menores do que $100 \mu \mathrm{m}$, ou seja, uma porcentagem considerada segura para as pulverizações (Tabela 5).

$\mathrm{O}$ coeficiente de uniformidade expressa à uniformidade do espectro das gotas pulverizadas. Espectro de gotas homogêneo tem valor deste coeficiente mais próximo de zero (VIANA et al., 2010). Neste estudo, os tratamentos Roundup Transorb ${ }^{\circledR}+$ Primóleo $^{\circledR}$ na dose de $3 \mathrm{~L}$ ha $^{-1}$ e Roundup Transorb ${ }^{\circledR}+$ Proof $^{\circledR}$ nas doses de 2,4 e 4,8 L $\mathrm{ha}^{-1}$ tiveram maior uniformidade no seu espectro de gotas em relação aos demais tratamentos, confirmado pelo menor valor do coeficiente de uniformidade (Tabela 5).

\section{Conclusões}

Chama-se atenção que as conclusões abaixo são baseadas em avaliações de misturas de herbicidas com água padrão cuja dureza total era de $20 \mathrm{mg} \mathrm{kg}^{-1}$ em equivalente de Carbonato de Cálcio:

1. Há incompatibilidade física dos herbicidas Primóleo $^{\circledR}$ e Proof ${ }^{\circledR}$ em mistura com Roundup Transorb ${ }^{\circledR}$ independente da agitação da calda.

2. É fundamental a agitação constante da calda fitossanitária antes e durante a aplicação para diminuir problemas de incompatibilidade entre os produtos.

3. O herbicida Proof ${ }^{\mathbb{B}}$ utilizado somente com a água possui $\mathrm{pH}$ próximo à neutralidade.

4. Os herbicidas avaliados de forma isolada ou em mistura têm valores de tensão superficial e ângulo de contato que proporcionam espalhamento na superfície do alvo e, consequentemente maior cobertura e controle do alvo visado.
5. O diâmetro das gotas pulverizadas varia de acordo com a mistura e a dose do herbicida avaliado.

6. Nas configurações do equipamento de aplicação (pressão, modelo de ponta e volume) descritas no trabalho, os herbicidas avaliados isolados ou em mistura tiveram percentual de deriva considerado seguro do ponto de vista ambiental.

7. Os herbicidas Roundup Transorb ${ }^{\circledR}+$ Proof $^{\circledR}$ na maior ou menor dose possuem espectro de gotas mais uniforme em relação aos demais tratamentos avaliados.

\section{Referências}

ABNT NBR 13875/2014. Agrotóxico - Avaliação de compatibilidade físico-química. Associação Brasileira de Normas Técnicas, 2014.

Arvidsson, T.; Bergström, L.; Kreuger, J. Spray drift as influenced by meteorological and technical factors. Pesticide Management Science, v. 67, n. 5, p. 586-598, 2011.

ASABE - American Society of Agricultural and Biological Engineers. Spray nozzle classification by droplet spectra. 2009. Disponível em: $<$ www.pubs.ext.vt.edu/442/442031/442-031_pdf.pdf $>$. Acesso em: 09 jun. 2017.

Costa, N.V.; Zobiole, L. H. S.; Scariot, C. A.; Pereira, G. R.; Moratelli, G. Glyphosate tolerant volunteer corn control at two development stages. Planta Daninha, v. 32, n. 4, p. 675 $-682,2014$.

Costa, L. L.; Silva, H. J. P. S.; Almeida, D. P.; Ferreira, M. C. Pontes, N. C. Droplet spectra and surface tension of spray solutions by biological insecticide and adjuvants. Engenharia Agrícola, v. 37, n. 2, p. 292-301, 2017a.

Costa, L .L.; Carneiro, A. L. C. G.; Souza, A. D. V.; Almeida, D. P.; Ferreira, M. C. Caracterização da aplicação com diferentes inseticidas e pontas de pulverização na cultura da soja. Revista Engenharia na Agricultura, v. 25, n. 2, p. 116-123, 2017 b.

Cunha, J. P. A. R.; Teixeira, M. M.; Coury, J. R.; Ferreira, L. R. Avaliação de estratégias para a redução da deriva de agrotóxicos em pulverizações hidráulicas. Planta Daninha, v. 21, n. 10 , p. $325-332,2003$.

Cunha, J. P. A. R.; Alves, G. S. Características físicoquímicas de soluções aquosas com adjuvantes de uso agrícola. Interciência, v. 34, n. 9, p. 655-659, 2009.

Dan, H. A.; Procópio, S. O.; Barroso, A. L. L.; Dan, L. G. M.; Oliveira Neto, A. M.; Guerra, N. Controle de plantas voluntárias de soja com herbicidas utilizados em milho. Revista Brasileira de Ciências Agrárias, v, 6, n. 2, p. 253257, 2011.

Decaro Júnior, S. T.; Ferreira, M. C.; Lasmar, O.; Campos, 
H. B. N. Relationship among variables of sprays applied at reduced volumes in a coffee plantation. Aspects of Applied Biology, v. 122, n. 1, p. 415-422, 2014.

Decaro Júnior, S. T.; Ferreira, M. C.; Lasmar, O. Physical characteristics of oily spraying liquids and droplets formed on coffee leaves and glass surfaces. Engenharia Agrícola, v. 35, n. 3, p. 588-600, 2015.

Gazziero, D. L. P. Misturas de agrotóxicos em tanques nas propriedades agrícolas do Brasil. Planta Daninha, v. 33, n. 1, p. 83-92, 2015.

Grigolli, J. F. J.; Gitti, D. C.; Lourenção, A. L. F. Controle de plantas de soja e supressão do capim em milho consorciado com Brachiaria ruziziensis. Arquivos do Instituto Biológico, v. 84, n. 1, p. 1-7, 2017.

Ikeda, F. S. Resistência de plantas daninhas em soja resistente ao glifosato. Informe Agropecuário, v. 34, n. 276, p. 1-8, 2013.

Marca, V.; Procópio, S. O.; Silva, A. G.; Volf, M. Chemical control of glyphosate-resistant volunteer maize. Revista Brasileira de Herbicidas, v. 14, n. 2, p. 103-110, 2015.

Murphy, G. Water $\mathbf{p H}$ and its effect on pesticides. Ontário: Ministry of Agriculture and Food. Disponível em: <www.gov.on.ca/OMAFRA/english/crops/hort/news/ grower/2004/08gn04a1.htm>. 2004. Acesso em: 10 jun 2018.

Nicolai, M.; Christoffoleti, P. J. Interações entre glyphosate e adubos foliares sobre parâmetros agronômicos do herbicida. Boletim Informativo da SBCPD, v. 15, p. 3943, 2007.

Oliveira, R. B.; Antuniassi, U. R.; Gandolfo, M. A. Spray adjuvant characteristics affecting agricultural spraying drift. Engenharia Agrícola, v. 35, n. 1, p. 109-116, 2015.

Petter, A. F.; Segate, D.; Almeida, F. A.; Neto, F. A.; Pacheco, L. P. Incompatibilidade física de misturas entre herbicidas e inseticidas. Planta Daninha, v. 30, n. 2, p. 449457, 2012.

Petter, A. F.; Segate, D.; Almeida, F. A.; Neto, F. A.; Pacheco, L. P. Incompatibilidade física de misturas entre inseticidas e fungicidas. Comunicata Scientiae, v. 4, n. 2, p. 129-138, 2013.

Petter, F. A.; Sima, V. M.; Fraporti, M. B.; Pereira, C. S.; Procópio, S. O.; Silva, A. F. Volunteer RR® corn management in roundup ready ${ }^{\circledR}$ soybean - corn succession system. Planta Daninha, v. 33, p. 119-128, 2015.

Queiroz, A. A.; Martins, J. A. S.; Cunha, J. P. A. R. Adjuvantes e qualidade da água na aplicação de agrotóxicos. Bioscience Journal, v. 24, n. 4, p. 8-19, 2008.
Takano, H. K.; Junior, R. S. O.; Constantin, J.; Biffe, D. F.; Franchini, L. H. M.; Braz, G. B. P.; Rios, F. A. Efeito da adição do 2,4-D ao glyphosate para o controle de espécies de plantas daninhas de difícil controle. Revista Brasileira de Herbicidas, v. 12, n. 1, p. 1-13, 2013.

Wanamarta, G.; Penner, D. Foliar absorption of herbicides. Weed Science, v. 4, p. 215-231, 1989.

Van Zyl, S. A.; Brink, J. C.; Calitz, F. J.; Coertze, S.; Fourie, P. H. The use of adjuvants to improve spray deposition and Botrytis cinerea control on Chardonnay grapevine leaves. Crop Protection, v. 29, n. 1, p. 58-67, 2010.

Viana, R. G.; Ferreira, L. R.; Ferreira, M. C.; Teixeira, M. M.; Rosell, J. R.; Tuffi Santos, L. D.; Machado, A. F. L. Distribuição volumétrica e espectro de gotas de pontas de pulverização de baixa deriva. Planta Daninha, v. 28, n. 2, p. 439-446, 2010.

Xu, L.; Zhu, H.; Ozkan, E.; Bagley, B.; Krause, C. R. Droplet evaporation and spread on waxy and hairy leaves associated with the type and concentration of adjuvants. Pest Management Science, v. 67, n. 7, p. 842-851, 2011. 